\title{
Left subclavian artery aneurysm secondary to syphilitic arteritis presenting with a right ischemic cerebellar infarction
}

\author{
Chih Ming Lin
}

No. 92, Sec.2, Chungshan North Rd. Taipei 112, Taiwan (R.O.C) Taipei, Taiwan

\begin{abstract}
Address for correspondence:

Dr. Chih Ming Lin,

No. 92, Sec.2, Chungshan

North Rd. Taipei 112 ,

Taiwan (R.O.C) Taipei, Taiwan

E-mail:submission_tw@

medcomasia.net

We report a 28-year-old male who presented with severe vertigo, vomiting, diplopia, clumsiness of right extremities. Physical examination revealed low reading of blood pressure in the left upper limb and evidence of mild left upper limb ischemia. On neurological examination he had right cerebellar ataxia. Computed tomography and magnetic resonance imaging, revealed an acute right cerebellar infarction in the anterior inferior cerebellar artery territory. Doppler ultrasonography revealed an aneurysm of the left subclavian artery. The patient was tested positive for serology of syphilis,
\end{abstract}

DOI: $10.4103 / 0028-3886.53279$
Key words: Subclavian aneurysm, ischemic infarction, syphilitic arteritis

\section{Introduction}

Neurological syndromes related to ischemic brain infarction can be lateralized and localized by good neurologic examination. However vascular distribution can be determined with mo derate to good reliability on the basis of physical examination alone. ${ }^{[1]}$ This report decribes a patients with right cerebellar ataxia due right cerebellar ischemic infarct and left subclavian aneurysm secondary syphilitic arteritis.

\section{Case Report}

A 28-year-old male presented in the emergency department with sudden onset severe vertigo, vomiting, double vision, and clumsiness in his right extremities. He also complained of a pulsatile mass above the left clavicle that had progressively enlarged over the past 2 weeks. He denied any history of trauma and his medical history was unremarkable. Physical examination revealed mild coldness of left uppe $r$ limb with cyanosis and low reading of blood pressure. A pulsatile swelling measuring $2 \mathrm{~cm}$ was noted in the left supra-clavicular fossa. On neurological examination he had right-sided dysmetria and ataxic gait, otherwise neurologic examination was normal.

Blood count and tests for protein-S, antithrombin-III, and total protein were normal. Rapid plasma reagin (RPR) and Treponema pallidum haemagglutination (TPHA) tests were positive. Serum fluorescent treponemal antibody absorption (FTA-AB) test confirmed the diagnosis of syphilis. Human immunodeficiency virus serology, cerebrospinal fluid (CSF) analsysi, and subsequent CSF Venereal Disease Research Laboratory (VDRL) tests were all negative.

Nonenhanced computed tomography (CT) brain imaging revealed hypodensity in the territory of the proximal right anterior inferior cerebellar artery (AICA) [Figure1]. Magnetic resonance imaging (MRI) using T1 fluid-attenuated inversion recovery (FLAIR), T2 FLAIR, and T2 weighted imaging (WI) confirmed acute infarction in the territory of the AICA [Figure 2]. There was restricted apparent diffusion on diffusion weighted imaging and parenchymal enhancement after contrast administration. MR-angiography, axial and 
sagittal source images, of the neck indicated evidence of a lobulated vascular lesion compressing the left subclavian artery [Figure 3]. Color-coded duplex Doppler sonography of the neck and vessels of the left arm revealed high-flow velocity in the left carotid system and low-flow velocity in the left carotid system with no diastolic dip in the distal subclavian artery suggesting a distal vessel obstruction or a reduction in vessel wall elasticity. Conventional angiography revealed a lobular aneurysm arising from the left subclavian artery downstream from the vertebral artery [Figure 4].

The patient was treated with clopidogrel, $75 \mathrm{mg}$ per day, and received standard benzathine penicillin G therapy (2 million units, intramuscular, weekly for 3 weeks). The aneurysm was treated with stent-grafting and embolization. At the follow-up visit, a physical examination revealed decreasing in size of the left supra-clavicular mass. Two years after the procedure, a duplex Doppler ultrasonography indicated normal blood flow in the left arm.

\section{Discussion}

This patient presented with right cerebellar ataxia and left subclavian aneurysm with evidence of left upper limb ischemia and low blood pressure reading in the left arm. One would have expected left cerebellar ataxia with the vascular pathology which he had, the clinical discrepancy. Emboli arising from the turbulent flow inside the left subclavian artery aneurysm was the most likely mechanism of infarction in our patient. The

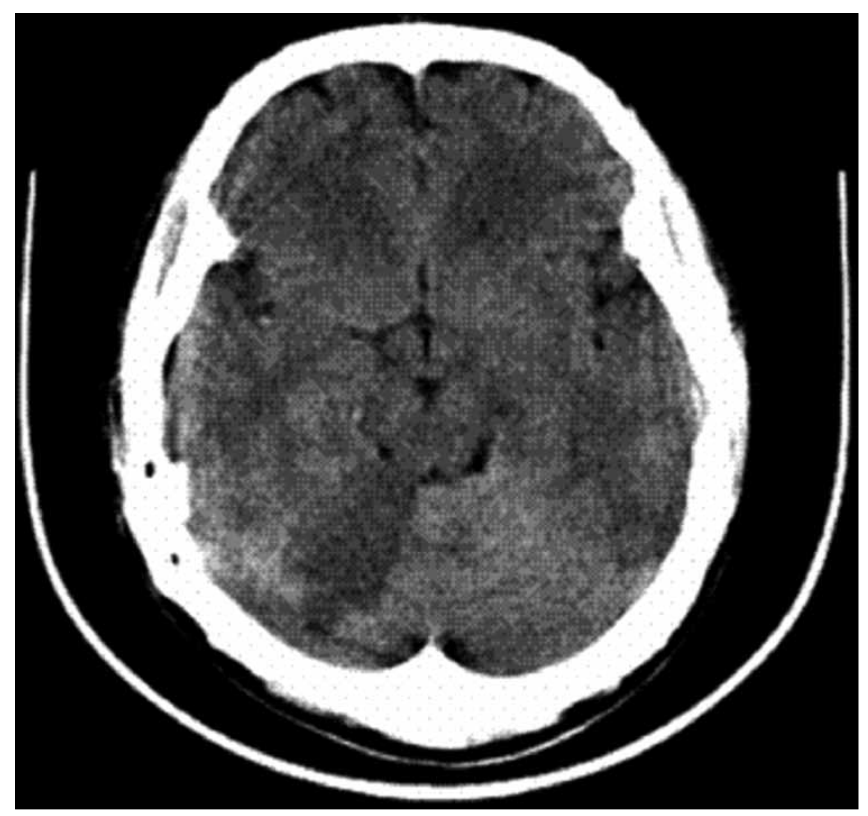

Figure 1: Noncontrast axial computed tomography scan showing hypodensity in the territory of the proximal anterior inferior cerebellar artery aneurysm was distal to the origin of the left vertebral artery and the infarct was in the right cerebellar hemisphere. The most plausible explanation is that emboli formed within the aneurysm might have gone retrogradly by the turbulent flow to the left vertebral artery, and then to the basilar artery, thus causing the infarction.

Syphilis is a chronic multisystem infection caused by the spirochete Treponema pallidum. About 10-12 million new infections are reported per year, ${ }^{[2]}$ and evidence suggests that the incidence of syphilis is increasing worldwide ${ }^{[3]}$ Tertiary syphilis can be associated with a constellation of symptoms including paresis, impaired memory and cognition, psychiatric disease, all of which can mistakenly be attributed to stroke. ${ }^{[1,4]}$ Neurosyphilis was common befor the penicillin era, but now it is uncommon as effective treatment is available. ${ }^{[1]}$

Several case reports have described association between syphilis and aneurysms, ${ }^{[5]}$ infarction, ${ }^{[6]}$ or both conditions ${ }^{[7]}$ In Taiwanese patients, atherosclerosis is the common cause of carotid occlusion, of the forty-four patients studied, only one patients had arterial occlusion due to syphilis. In this series 11 patients had Takayasu's arteritis. ${ }^{[8]} \mathrm{It}$ is very difficult to diffentiate between occlusion of an infectious etiology (syphilis) and non-infectious arterial occlusion $^{[9]}$ In our patient, negative CSF VDRL test and

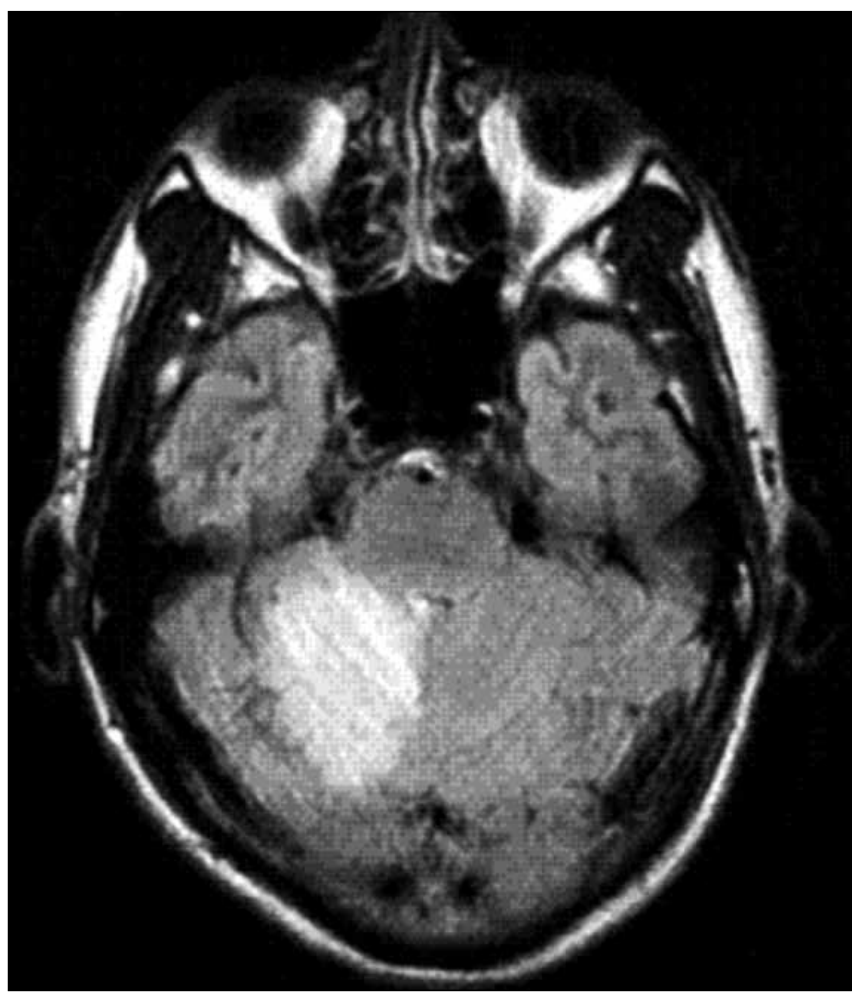

Figure 2: Fluid-attenuated inversion recovery imaging in the axial plane showing acute infarction in the territory of the AICA 


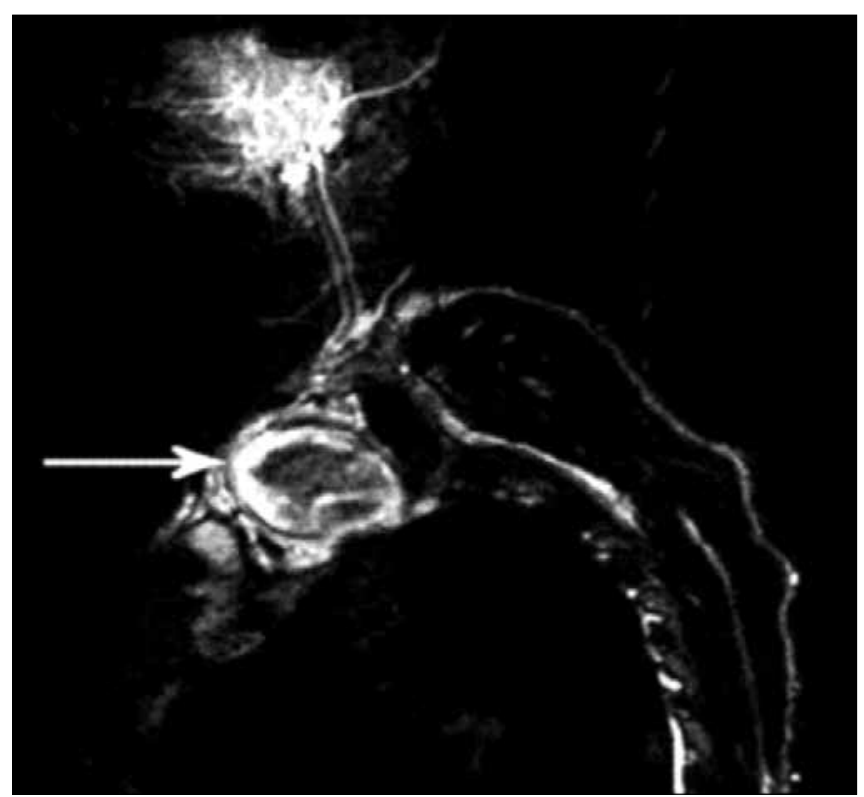

Figure 3: Magnetic resonance imaging of the lower left region of the neck performed in sagittal section after contrast enhancement showing a heterogenously hypo- and hyperintense round mass lesion with contrast eenhancement in the inferior portion of the lesion with compression of the left subclavian artery is noted

normal CSF biochemistry suggest the clinical syndrome was related to syphilitic arteritis and aneurysm and not to neurosyphilis. This case also illustrates the importance of work-up for syphilis in young stroke.

\section{References}

1. Goldstein LB, Simel DL. Is this patient having a stroke? JAMA 2005;293: 2391-402

2. French P. Syphilis. Brit Med J 2007;334:143-7.

3. Chakraborty R, Luck S. Syphilis is on the increase: The implications for child health. Arch Dis Child 2008;93:105-9.

4. Barrett AM. Is it Alzheimer's disease or something else? 10 disorders that may feature impaired memory and cognition. Postgrad Med $2005 ; 117: 47-53$.

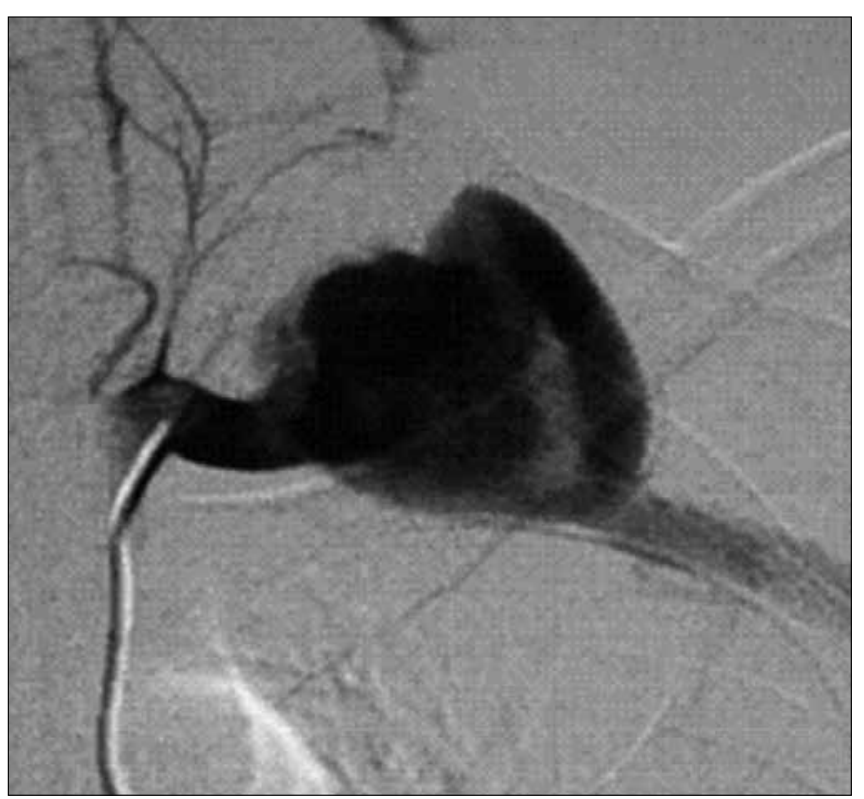

Figure 4: Conventional angiography revealed a lobular aneurysm arising from the left subclavian artery downstream of the left vertebral artery orifice

5. Asano M, Hiroshi O, Hanafusa Y, Kazuma H, Tanji M. Intramural hematoma and thoracic aortic aneurysm with syphilis. J Thor Cardivasc Surg 2007;133:1085-6

6. Flint AC, Liberato BB, Anziska Y, Schantz-Dunn J, Wright CB Meningovascular syphilis as a cause of basilar artery stenosis. Neurology 2005;64:391-2

7. Kellett MW, Young GR, Fletcher NA. Paraparesis due to syphilitic aortic dissection. Neurology 1997;48:221-3.

8. Tsai CF, Jeng JS, Lu CJ, Yip PK. Clinical and ultrasonographic manifestations in major causes of common carotid artery occlusion. J Neuroimaging 2005;15:50-6.

9. Tavora F, Burke A. Review of isolated ascending aortitis: Differential diagnosis, including syphilitic, Takayasu's and giant cell arteritis. Pathology 2006;38:302-8.

Accepted on 23-02-2009

Source of Support: Nil, Conflict of Interest: None declared. 